\author{
Military Technical College \\ Kobry El-Kobbah, \\ Cairo, Egypt
}

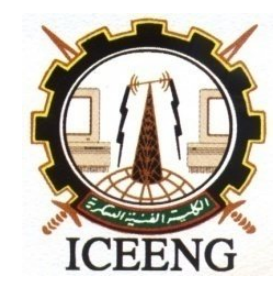

\author{
$7^{\text {th }}$ International Conference \\ on Electrical Engineering \\ ICEENG 2010
}

\title{
Observability Analysis and Kalman Filter Applications for Error Estimation of SDINS Ground Alignment
}

\author{
By \\ I. I. Arfaa $^{* *}, \quad$ Y. Z. Elhalwagy ${ }^{* *} \quad$ R. A. Elbordany ${ }^{* *}, \quad$ and $\quad$ M. Deab ${ }^{* *}$
}

\section{$\underline{\text { Abstract: }}$}

A systematic analysis of the observability of a strap down inertial navigation system (SDINS) in ground alignment with Bar-Itzhack and Berman's error model is presented. It is shown that the unobservable states are separately contained in two de-coupled subspaces. The constraints on the selection of unobservable states are discussed. An estimation algorithm, which is derived fully from the horizontal velocity outputs for computing the misalignment angles, is provided. It reveals that the azimuth error can be entirely estimated from the estimates of leveling error and leveling error rate, without using gyro output signals explicitly. Moreover, estimate of the strap down inertial navigation systems errors are presented using appropriate Kalman filter design.

\section{Keywords:}

Inertial Sensors Alignment, SDINS, KF, Observability Analysis

\footnotetext{
** Egyptian Armed Forces

** Egyptian Armed Forces

** Egyptian Armed Forces

${ }^{* *}$ Egyptian Armed Forces
} 


\section{Introduction:}

Initial misalignment is one of the major error sources of inertial navigation systems (INS). For terrestrial navigation, the initial alignment errors will affect the system error not only in the attitude indication but also in the velocity and position information [1-3]. Therefore, prior to normal navigation, alignment process must be performed to determine the orientation of the platform axes with respect to the navigation coordinate frame. One method for obtaining the initial angular orientation is through the use of an external reference by optical means. However, this method is very limited to operational environment. Alternatively, for most ground-based applications, a self-contained alignment method known as gyro-compassing [2-7] provides another operational approach. As a general rule, gyro-compassing consists of two phases, that is, leveling and azimuth alignment. The basic principle of gyro-compassing consists of feeding signals proportional to the accelerometer outputs and/or velocity error outputs to the appropriate level gyros and azimuth gyro.

The purpose of initial alignment process is to drive the misalignment angles to zero. Unfortunately, this goal can never be achieved in a practical system. This drawback is deduced from the sensor errors, which cannot be compensated ideally. Theoretically, the basic difficulty associated with the selfalignment technique is that the system is not completely observable. The determination of unobservable states during initial alignment process is very important in consideration of system performance. Generally, the system state variable can be transformed into the observable canonical form. It means that the observable part and unobservable part can be separated intentionally. However, the choice of observable (unobservable) states is certainly not unique since the numbers of transformations are innumerable [8]. This problem is analyzed by different approaches as the methods presented in $[2,3]$, and results are obtained.

In the literature, it has been showed that the determination of unobservable states was a part of physical judgments. This interesting problem of selection of the suitable unobservable states motivates us to investigate the observability of INS during ground alignment phase of operation.

In this paper, the observability of a linearized SDINS error model of a stationary vehicle is examined. The horizontal velocity outputs of INS are the system measurements. It has been found that the unobservable states, which are distributed in two decoupled subspaces, can be systematically determined. Moreover, the leveling errors can be estimated from the system measurements and their first derivatives. However, to estimate the azimuth error, the second derivatives of system measurement are needed. It is equivalent to state that the estimation of azimuth error can be obtained from the estimates of leveling error and leveling error rate.

This phenomenon may facilitate the designing of filters for leveling and azimuth alignment simultaneously, without using gyro outputs explicitly. On the other hand, the error covariance matrix of the Kalman filter could be a good performance index for the degree of observability of a system. If the estimation of a state by Kalman filter is convergent, the state will be observable. If the estimation of a state is dis-convergent, the state will be unobservable. The faster the convergence rate is the higher the degree of observability of the state.

\section{$\underline{\text { 2. Ground Alignment Error Model: }}$}

In order to look into the behavior of an inertial navigator, a proper error model is derived. It is well known that the description of the INS error propagation using a linearized error model is quite a good approximation. The characteristics of SDINS can be derived from the linear model. Many different error models can be found in the literature. For analyses purposes, Bar-Itzhack and Berman's [9] derivation of the error model for INS in ground alignment is adopted. 
In this study, the navigation frame is the local-level East (E), North (N), and Up (U) coordinate system. Since the coupling of the vertical channel with the horizontal channels is very weak, the vertical channel can be ignored. When the initial alignment process is accomplished at a fixed ground base where the geographic position is known precisely then the gravity error and the position error state can be ruled out. Moreover, the system is nearly stationary and hence the coriolis acceleration can also be ignored. Under these assumptions, the error dynamics including the horizontal velocity errors and the attitude errors can be made considerably simple. In this case, the INS ground alignment error model can be written as [10]

$$
\left[\begin{array}{l}
\delta \dot{v}_{x} \\
\delta \dot{v}_{y} \\
\dot{\psi}_{x} \\
\dot{\psi}_{y} \\
\dot{\psi}_{z}
\end{array}\right]=\left[\begin{array}{ccccc}
0 & 2 \Omega_{U} & 0 & -g & 0 \\
-2 \Omega_{U} & 0 & g & 0 & 0 \\
0 & 0 & 0 & \Omega_{U} & -\Omega_{N} \\
0 & 0 & -\Omega_{U} & 0 & \Omega_{E} \\
0 & 0 & \Omega_{N} & -\Omega_{E} & 0
\end{array}\right]\left[\begin{array}{l}
\delta v_{x} \\
\delta v_{y} \\
\psi_{x} \\
\psi_{y} \\
\psi_{z}
\end{array}\right]+\left[\begin{array}{c}
\nabla_{x} \\
\nabla_{y} \\
\varepsilon_{x} \\
\varepsilon_{y} \\
\varepsilon_{z}
\end{array}\right]
$$

Where $\delta v$ and $\psi$ represent the velocity error and attitude error, respectively; $\nabla$ and $\varepsilon$ represent, respectively, the generalized accelerometer error and the generalized gyro drift rate [10]; $g$ is the local gravity; $\Omega$ represent the Earth rate; $\phi$ is the local geographic latitude angle. The subscripts $\mathrm{x} \mathrm{y}$ and $\mathrm{z}$ denote the corresponding components in the East-North-Up navigation coordinate system. Where;

$\Omega_{U}=\Omega \sin \phi, \Omega_{\mathrm{N}}=\Omega \cos \phi, \Omega_{\mathrm{E}}=0$.

Rewrite (1) in compact notation

$\dot{x}^{\prime}=A^{\prime} x^{\prime}+b$

Where variables $x^{\prime}, A^{\prime}$, and $b$ are identified with their counterparts in (1). It is reasonable and practical to assume the generalized accelerometer errors and gyro drift rates as constant in ground alignment phase. Hence the sensor errors can be modeled as:

$$
[\dot{b}]=\left[\begin{array}{l}
\dot{\nabla} \\
\dot{\varepsilon}
\end{array}\right]=0
$$

Where $\nabla=\left[\nabla_{x}, \nabla_{y}\right]^{T}$ represents the generalized accelerometer error vector and $\varepsilon=\left[\varepsilon_{x}, \varepsilon_{y}, \varepsilon_{z}\right]^{T}$ represents the generalized gyro drift rate vector.

Combing (2) and (3) yields:

$$
\left[\begin{array}{c}
\dot{x^{\prime}} \\
\dot{b}
\end{array}\right]=\left[\begin{array}{cc}
A^{\prime} & I \\
0 & 0
\end{array}\right]\left[\begin{array}{c}
x^{\prime} \\
b
\end{array}\right]
$$

Where $I$ is the identity matrix. This linear dynamic error model can also be expressed in compact notation as:

$$
x=A x
$$

Where $x^{T}=\left[x^{\prime}, \nabla, \varepsilon\right]^{T}$ and the definition of $A$ is obvious from (4)

Finally, we consider the outputs of INS horizontal velocity components as the system measurements, namely,

$$
z=\left[z_{1}, z_{2}\right]^{T}=\left[\delta v_{x}, \delta v_{y}\right]^{T}
$$

Then the relationship between the measurements and the system $A^{\prime}$ in (2) can be written as: 
$z=C^{\prime} x^{\prime}$

Where

$$
C^{\prime}=\left[\begin{array}{lllll}
1 & 0 & 0 & 0 & 0 \\
0 & 1 & 0 & 0 & 0
\end{array}\right]
$$

Similarly, the relationship between the measurements and the system $A$ in (5) can be written as: $z=C x$

Where

$$
C=\left[\begin{array}{llllllllll}
1 & 0 & 0 & 0 & 0 & 0 & 0 & 0 & 0 & 0 \\
0 & 1 & 0 & 0 & 0 & 0 & 0 & 0 & 0 & 0
\end{array}\right]
$$

Now, the observability of the system $A^{\prime}$ and the system $A$ can be analyzed.

\section{$\underline{\text { 3. Observability Test }}$}

The necessary condition for the observable system is that the observability test matrix is full rank. If the rank of the observability test matrix is equal to the order of the system then the system is completely observable. On the contrary, if the system is not completely observable, the number of the unobservable states is the difference between the order of the system and the rank of the observability test matrix.

In general, the observability test matrix for the system matrix $A$ with measurement $C$ can be expressed as:

$$
O=\left[\begin{array}{c}
C^{\prime} \\
C^{\prime} A^{\prime} \\
\cdot \\
C^{\prime} A^{\prime n-1}
\end{array}\right]
$$

Where $n$ is the order of the system. The observability test matrix for the system $A^{\prime}$ with measurement matrix $C^{\prime}$ can be written as:

$$
Q^{\prime}=\left[\begin{array}{c}
C^{\prime} \\
C^{\prime} A^{\prime} \\
\cdot \\
C^{\prime} A^{\prime 4}
\end{array}\right]
$$

It is easily seen that the rank of $Q^{\prime}$ is 5 , which is equal to the order of the system $A^{\prime}$. Thus the matrix $Q^{\prime}$ is full rank. So, the system $A^{\prime}$ is completely observable. It implies that if the sensor errors are fully compensated, the estimation problem during alignment process can be automatically solved [8].

For the system $A$ with measurement matrix $C$, the observability test can be written as:

$Q=\left[\begin{array}{c}C \\ C A \\ \cdot \\ C A^{9}\end{array}\right]$

It can be found that the rank of $Q$ is 7 which, is smaller than the system order 10 . Thus the matrix $Q$ is not full rank. So, the system $A$ is not completely observable and the estimation becomes an unsolvable problem with three unobservable states. It is clear that the observability loss in system $A$ is generated by augmentation from (2)-(5). It implies that the system can be made observable if the dynamics of the sensor errors are ignored. However, this assumption is practically weak. The 
determination of the unobservable states is now a key problem for estimation in alignment and calibration phase of operation.

Since the system measurements are observable by definition, states $\delta v_{x}$ and $\delta v_{y}$ are undoubted observable. For convenience, let's define the following:

$$
\begin{aligned}
& x_{1}=\left[\delta v_{x}, \delta v_{y}\right]^{T} \\
& x_{2}=\left[\psi_{x}, \nabla_{y}, \varepsilon_{y}, \varepsilon_{z}\right]^{T} \\
& x_{3}=\left[\psi_{y}, \psi_{z}, \nabla_{x}, \varepsilon_{x}\right]^{T}
\end{aligned}
$$

And

$$
\begin{aligned}
& y_{1}=\left[\begin{array}{l}
z_{1} \\
z_{2}
\end{array}\right]=\left[\begin{array}{l}
\delta \mathrm{v}_{\mathrm{x}} \\
\delta \mathrm{v}_{\mathrm{y}}
\end{array}\right] \\
& y_{2}=\left[\begin{array}{c}
z_{2}+2 \Omega_{U} z_{1} \\
\ddot{z}+4 \Omega_{U}^{2} z_{1} \\
z_{2}^{(i i i)}-8 \Omega_{U}^{3} z_{1} \\
z_{1}^{(i v)}-16 \Omega_{U}^{4} z_{1}
\end{array}\right] \\
& y_{3}=\left[\begin{array}{c}
z_{1}-2 \Omega_{U} z_{2} \\
z_{2}+4 \Omega_{U}^{2} z_{2} \\
z_{1}^{(i i i)}+8 \Omega_{U}^{3} z_{2} \\
z_{2}^{(i v)}-16 \Omega_{U}^{4} z_{2}
\end{array}\right]
\end{aligned}
$$

Since the rank of a matrix is invariant under elementary row operation, the observability associated with the matrix $Q,(13)$ is equivalent to the solvability of the following:

$$
\left[\begin{array}{l}
y_{1} \\
y_{2} \\
y_{3}
\end{array}\right]=\left[\begin{array}{ccc}
I & 0 & 0 \\
0 & Q_{2} & 0 \\
0 & 0 & Q_{3}
\end{array}\right]\left[\begin{array}{l}
x_{1} \\
x_{2} \\
x_{3}
\end{array}\right]
$$

Where $I$ is the identity matrix.

$$
Q_{2}=\left[\begin{array}{cccc}
g & 1 & 0 & 0 \\
3 g \Omega_{U} & 2 \Omega_{U} & -g & 0 \\
-7 g \Omega_{U}^{2}-g \Omega_{N}^{2} & -4 \Omega_{U}^{2} & 3 g \Omega_{U} & -g \Omega_{N} \\
-15 g \Omega_{U}^{3}-3 g \Omega_{N}^{2} \Omega_{U} & -8 \Omega_{U}^{3} & 7 g \Omega_{U}^{3} & -3 g \Omega_{N} \Omega_{U}
\end{array}\right]
$$

And

$$
Q_{3}=\left[\begin{array}{cccc}
-g & 0 & 1 & 0 \\
3 g \Omega_{U} & -g \Omega_{N} & -2 \Omega_{U} & g \\
7 g \Omega_{U}^{2} & -3 g \Omega_{U} \Omega_{N} & -4 \Omega_{U}^{2} & 3 g \Omega_{U} \\
-15 g \Omega_{U}^{3}-g \Omega_{N}^{2} \Omega_{U} & 7 g \Omega_{N} \Omega_{U}^{2}+g \Omega_{N}{ }^{3} & -8 g \Omega_{U}^{3} & -7 g \Omega_{U}^{2}-g \Omega_{N}^{2}
\end{array}\right]
$$

From equation (20), the observability of the system can be determined by the solvability of three decoupled matrix equations. It is obvious that $x_{1}$ is observable. Hence the three unobservable states must reside in $x_{2}$ and $x_{3}$ which are, respectively, governed by: 


$$
\begin{aligned}
& y_{2}=Q_{2} x_{2} \\
& y_{3}=Q_{3} x_{3}
\end{aligned}
$$

It is easily found that when the system is not at the Earth pole, $\Omega_{N} \neq 0$.

We can notice that the first column of $Q_{2}$ is a linear combination of other three columns. Then, from Equ. (21), the rank of $Q_{2}$ is 3 which is one less than the order of $Q_{2}$. Therefore, only one unobservable state can be chosen from the components of $x_{2}$.

Similarly, because the first column of $Q_{3}$ is a linear combination of the third and fourth columns, the second column of $Q_{3}$ is equal to the fourth column times $\Omega_{N}$. From Equ. (22), the rank of $Q_{3}$ is 2 . Thus, there are two unobservable states contained in $x_{3}$. By observation, $\psi_{z}$ and $\varepsilon_{x} / \Omega_{N}$ have the same effect on the measurement derivatives $y_{3}$. Therefore, Equ. (24) Can be written as:

$$
y_{3}=\left[\begin{array}{ccc}
-g & 0 & 1 \\
3 g \Omega_{U} & -g \Omega_{N} & -2 \Omega_{U} \\
7 g \Omega_{U}^{2} & -3 g \Omega_{N} \Omega_{U} & -4 \Omega_{U}^{2} \\
-15 g \Omega_{U}^{3}-g \Omega_{N}^{2} \Omega_{U} & 7 g \Omega_{N} \Omega_{U}^{2}+g \Omega_{N}^{3} & -8 \Omega_{U}^{3}
\end{array}\right]\left[\begin{array}{c}
\psi_{y} \\
\psi_{U}+\frac{\varepsilon_{x}}{\Omega_{N}} \\
\nabla_{x}
\end{array}\right]
$$

Which shows that only one of $\psi_{z}$ and $\varepsilon_{x}$ can be observed at a time. However, they can be chosen as unobservable states simultaneously. Thus $\psi_{y}$ and $\nabla_{x}$ must be observable. Note that when the system is located at the Earth pole, $\Omega_{N}=0$, both the fourth column of $Q_{2}$ and second column of $Q_{3}$ are zero. Then both $\varepsilon_{z}$ and $\psi_{z}$ are definitely unobservable. That is why the INS cannot be self-aligned at the pole. In this case, the rank of $Q_{2}$ is reduced to 2 and the rank of $Q_{3}$ is unchanged.

\section{Estimation of Misalignment Angles}

The objective of initial alignment is to drive the misalignment angles $\psi_{x}, \psi_{y}$ and $\psi_{z}$ to zero or as small as possible. It is necessary that these states be all observable. From the above analysis we found that $\nabla_{x}$ and $\varepsilon_{x}$ in $x_{3}$ are inevitable unobservable. Then, only one unobservable state can be chosen from the components of $x_{2}$. Theoretically, the choice is arbitrary except $\psi_{x}$. However, in order to achieve better accuracy, the unobservable state must be selected carefully. Intuitively, from the first two columns of $Q_{2}$, it is obvious that $\psi_{x}$ and $\nabla_{y} / g$ are strongly coupled. Besides, if we do not choose $\nabla_{y}$ as the unobservable state, more time derivatives of measurements are needed to compute the estimation which of $\psi_{x}$. That causes poor estimation, which should be avoided in practice. Therefore, the best choice of the unobservable states are $\nabla_{x}, \nabla_{y}$ and $\varepsilon_{x}$ for the system in ground alignment process. In this case, both $\varepsilon_{y}$ and $\varepsilon_{z}$ can also be estimated for the purpose of calibration. Once the unobservable states have been selected, we can engage in designing an estimation algorithm for computing the estimates of misalignment angles.

Combing the first equation of (23) and the first two equations of (25), we have

$$
\begin{gathered}
\dot{z}_{2}+2 \Omega_{U} z_{1}=g \psi_{x}+\nabla_{y} \\
\dot{z}_{1}-2 \Omega_{U} z_{2}=-g \psi_{y}+\nabla_{x}
\end{gathered}
$$




$$
\ddot{z}_{2}+4 \Omega_{U}^{2} z_{2}=-3 g \Omega_{U} \Omega_{N}+g \Omega_{N}\left(\psi_{z}+\frac{\varepsilon_{x}}{\Omega_{N}}\right)+2 \Omega_{U} \nabla_{x}
$$

Substituting (6) into the above equations and solving for misalignment angles, yields:

$$
\begin{aligned}
& \psi_{x}=\frac{1}{g}\left(\delta \dot{v}_{y}+2 \Omega_{U} \delta v_{x}-\nabla_{y}\right) \\
& \psi_{y}=-\frac{1}{g}\left(\delta \dot{v}_{x}+2 \Omega_{U} \delta v_{y}-\nabla_{x}\right) \\
& \psi_{z}=\frac{1}{g \Omega_{N}}\left(\delta \dot{v}_{y}-3 \Omega_{U} \delta \dot{v}_{x}-2 \Omega_{U}^{2} \delta v_{y}+\Omega_{U} \nabla_{E}\right)-\frac{\varepsilon_{x}}{\Omega_{N}}
\end{aligned}
$$

Since we have chosen $\nabla_{x}, \nabla_{y}$ and $\varepsilon_{x}$ as unobservable states, the best estimates can be obtained by sitting these unobservable states to zero, i.e.,

$$
\begin{aligned}
& \hat{\psi}_{x}=\frac{1}{g}\left(\delta \dot{v}_{y}+2 \Omega_{U} \delta v_{x}\right) \\
& \hat{\psi}_{y}=-\frac{1}{g}\left(\delta \dot{v}_{x}+2 \Omega_{U} \delta v_{y}\right) \\
& \hat{\psi}_{z}=\frac{1}{g \Omega_{N}}\left(\delta \ddot{v}_{y}-3 \Omega_{U} \delta \dot{v}_{x}-2 \Omega_{U}^{2} \delta v_{y}\right)
\end{aligned}
$$

These equations show that the leveling errors, $\psi_{E}$ and $\psi_{N}$, can be estimated from the system measurements and their first time derivatives, and the azimuth error, $\psi_{U}$, can be estimated from the measurements and their time derivatives up to second order.

It is evident, from (32-34) and (29-31) that the errors in the estimation are:

$$
\left[\begin{array}{c}
\Delta \psi_{x} \\
\Delta \psi_{y} \\
\Delta \psi_{z}
\end{array}\right]=\left[\begin{array}{c}
\hat{\psi}_{x}-\psi_{x} \\
\hat{\psi}_{y}-\psi_{y} \\
\hat{\psi}_{z}-\psi_{z}
\end{array}\right]=\left[\begin{array}{c}
\frac{\nabla_{y}}{g} \\
\frac{-\nabla_{x}}{g} \\
\frac{\varepsilon_{x}}{\Omega_{N}}-\frac{\nabla_{x}}{g} \tan \phi
\end{array}\right]
$$

This result is identical with the accuracy that is often shown in the self-alignment schemes $[2,7,9]$. The above equation shows that the leveling estimation errors are caused by the accelerometer errors. The east gyro drift rate and the north leveling error cause the azimuth estimation error. Both of them are latitude dependent [8].

Finally, differentiating (33), yields:

$$
\dot{\hat{\psi}}_{x}=\frac{1}{g}\left(\delta \ddot{v}_{y}-2 \Omega_{U} \delta \dot{v}_{x}\right)
$$

Substituting (32) and (36) into (34), it can be found that:

$$
\hat{\psi}_{z}=\frac{1}{\Omega_{N}}\left(\dot{\hat{\psi}}_{x}+\Omega_{U} \hat{\psi}_{y}\right)
$$

Which shows that the azimuth error can be computed from the estimates of the leveling error about north axis and the leveling error rate about east axis. Note that the estimation of azimuth error dose not explicitly depend on gyro output signal. This phenomenon can be used in an alternate filter design for leveling and azimuth alignment simultaneously [11-13]. 


\section{Kalman Filter Design}

In this work we have modified Bar-Itzhack and Berman's inertial navigation system error model [14]. The SDINS stationary error model augmented with sensor errors can be written as:

$$
\begin{aligned}
& {\left[\begin{array}{c}
\dot{x}_{a} \\
\dot{x}_{b}
\end{array}\right]=\left[\begin{array}{cc}
F & I_{5 \times 5} \\
O_{5 \times 5} & O_{5 \times 5}
\end{array}\right]\left[\begin{array}{l}
x_{a} \\
x_{b}
\end{array}\right]+\left[\begin{array}{c}
W \\
O_{5 \times 1}
\end{array}\right]=A x+W} \\
& W \sim N(0, Q)
\end{aligned}
$$

Where $I$ is the identity matrix with the order as in (35), and the state vectors are construct as follows:

$$
\begin{aligned}
& x_{a}=\left[\begin{array}{lllll}
\delta v_{x} & \delta v_{y} & \psi_{x} & \psi_{y} & \psi_{z}
\end{array}\right]^{T} \\
& x_{b}=\left[\begin{array}{lllll}
\nabla_{x} & \nabla_{y} & \varepsilon_{x} & \varepsilon_{y} & \varepsilon_{z}
\end{array}\right]^{T}
\end{aligned}
$$

The system dynamic matrix $F$ and the process noise vector $\vec{W}$ is define respectively as follows:

$$
\begin{aligned}
& F=\left[\begin{array}{ccccc}
0 & 2 \Omega_{U} & 0 & -g & 0 \\
-2 \Omega_{U} & 0 & g & 0 & 0 \\
0 & 0 & 0 & \Omega_{U} & -\Omega_{N} \\
0 & 0 & -\Omega_{U} & 0 & \Omega_{E} \\
0 & 0 & \Omega_{N} & -\Omega_{E} & 0
\end{array}\right] \\
& \vec{W}=\left[\begin{array}{lllll}
W_{v_{x}} & W_{v_{y}} & W_{\psi_{x}} & W_{\psi_{y}} & W_{\psi_{z}}
\end{array}\right]^{T}
\end{aligned}
$$

The measured signals during the stationary alignment are the horizontal velocity errors. Thus consider the observation model as follows:

$$
z=\left[\begin{array}{llllllllll}
1 & 0 & 0 & 0 & 0 & 0 & 0 & 0 & 0 & 0 \\
0 & 1 & 0 & 0 & 0 & 0 & 0 & 0 & 0 & 0
\end{array}\right]\left[\begin{array}{c}
x_{a} \\
x_{b}
\end{array}\right]+\left[\begin{array}{l}
\Gamma_{1} \\
\Gamma_{2}
\end{array}\right]=C x+\Gamma \quad \Gamma \sim N(0, R)
$$

In terms of the error model and the observation model of SDINS, the discrete Kalman filtering equations are formulated as follows:

$$
\begin{aligned}
& \hat{x}_{k, k-1}=A_{k, k-1} x_{k-1} \\
& \hat{x}_{k}=\hat{x}_{k, k-1}+K_{k}\left[z_{k}-C_{k} \hat{x}_{k, k-1}\right] \\
& K_{k}=P_{k, k-1} C_{k}^{T}\left[C_{k} P_{k, k-1} C_{k}^{T}+R_{k}\right]^{-1} \\
& P_{k, k-1}=A_{k, k-1} P_{k-1} A_{k, k-1}^{T}+Q_{k-1} \\
& P_{k}=\left[I-K_{k} C_{k}\right] P_{k, k-1}\left[I-K_{k} C_{k}\right]^{T}+K_{k} R_{k} K_{k}^{T}
\end{aligned}
$$

Where $A_{k, k-1}$ is the discrete state transformation matrix (system matrix), $P_{k, k-1}$ is the covariance matrix representing errors in the state estimates, $P_{k}$ is a covariance matrix representing errors in the state estimates (i.e., diagonal elements represent variance of true state minus estimated state)after an update, $Q_{k}$ is the discrete process-noise matrix, $R_{k}$ is the measurement process-noise matrix and $K_{k}$ is the Kalman filter gains.

The initial value $x(0)$ of state $x$ is chosen as zero. $P_{1}(0), Q$ and $R$ for a medium accuracy SINS are chosen. The initial misalignment angles $\psi_{x}, \psi_{y}$ and $\psi_{z}$ is chosen as $1^{0}$, respectively. The constant and 
random biases of each accelerometer are chosen as $100 \mu \mathrm{g}$ and $10 \mu \mathrm{g}$, respectively, and the constant and random drifts of each gyro are chosen as $0.02^{0} / h$ and $0.01^{\circ} / h$, respectively. The measuring of two horizontal error velocities $\delta_{v}$ is $0.5 \mathrm{~m} / \mathrm{s}$ and measuring process-noise chosen as $\sigma_{\delta v}=.05 \mathrm{~m} / \mathrm{s}$. The local geographic latitude of SDINS place is $30^{\circ}$.

\section{Simulation Results}

Simulation results illustrate that among three misalignment angles, the two leveling misalignment angles $\psi_{x}$ and $\psi_{y}$ can be estimated effectively, the estimation error of $\psi_{x}$ and $\psi_{y}$ converge quickly, but the estimation error of state $\psi_{z}$ is found to converge slowly. The alignment time of $\psi_{z}$ is about 260 sec, but the convergence of estimation errors of $\psi_{x}$ and $\psi_{y}$ only needs about $50 \mathrm{sec}$, respectively. Table (1) shows the different convergence rates of $\psi_{x}, \psi_{y}$ and $\psi_{z}$. Also, it shows how the Kalman filter work precisely after discarding the three unobservable states $\nabla_{x}, \nabla_{y}, \varepsilon_{x}$.

Figs. $(1,2)$ show the different convergence rates of $\psi_{x}, \psi_{y}$ and $\psi_{z}$. In accuracy of estimation, the steady state value of $\psi_{z}$ is about $-6.0473^{\prime}$. It is due to unobservable state $\varepsilon_{x}$, but the estimation accuracy of $\psi_{x}$ and $\psi_{y}$ is very high about $20^{\prime \prime}$ approximately.

Table (1)

\begin{tabular}{|c|c|c|c|}
\hline & $\Delta \psi_{x}[\mathrm{sec}]$ & $\Delta \psi_{y}[\mathrm{sec}]$ & $\Delta \psi_{z}[\mathrm{sec}]$ \\
\hline 10 states & 19.7717 & -20.6612 & -362.8407 \\
\hline 7 states & 20.3962 & -21.4587 & -348.0337 \\
\hline
\end{tabular}




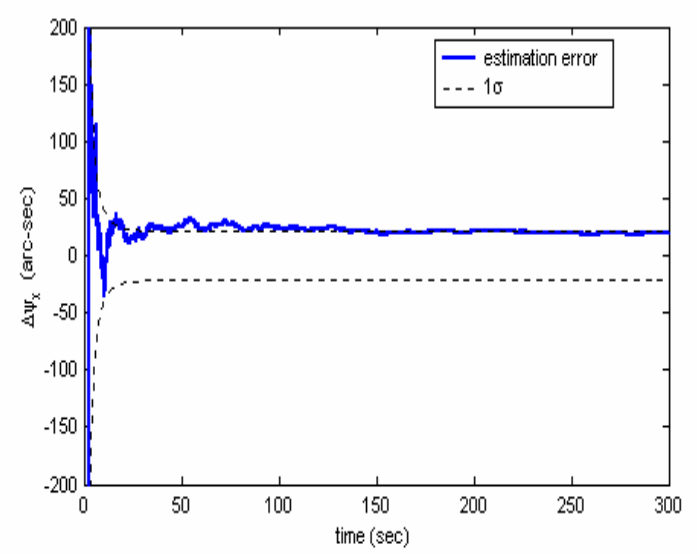

Figure (2): Estimation of gyro drift 


\section{Conclusions:}

Based upon Bar-Itzhack and Berman's SINS error model, the observability of INS operating in ground alignment phase is analyzed. It is realized that the unobservable states are induced by the augmentation of sensor errors. In general, there are three unobservable states with one contained in $x_{2}=\left[\psi_{x}, \nabla_{y}, \varepsilon_{y}, \varepsilon_{z}\right]^{T}$ and two contained in $x_{3}=\left[\psi_{y}, \psi_{z}, \nabla_{x}, \varepsilon_{x}\right]^{T}$. The selection of unobservable states from $x_{2}$ is arbitrary. However, the unobservable states in $x_{3}$ cannot be observed at the same time.

When the system is located at the Earth pole, the number of unobservable states becomes 4 among which $\psi_{z}$ and $\varepsilon_{x}$ is definitely unobservable states. The determination of the unobservable states is dependent upon mission requirements. For the purpose of alignment, the best choice of unobservable states are $\nabla_{x}, \nabla_{y}$ and $\varepsilon_{x}$. An estimation algorithm has been derived for aligning the SINS on ground stationary base. It shows that the leveling errors can be estimated from the measured velocity outputs and their first derivatives. While the second time derivative of North-velocity component is needed in estimating the azimuth misalignment angle. Furthermore, the estimated azimuth misalignment has been found proportional to the estimates of the leveling error about the north axis and the leveling error rate about the east axis. This property is useful for designing alternate filter algorithm for leveling and azimuth alignment.

In addition, the major motivation for the observability analysis was the correspondence between this analysis and the prediction of the estimability of the system. The estimability of the SINS during ground alignment was checked using appropriate Kalman filter design for the system. That is, we considered the real SINS error model, which is stochastic. That model is obtained when we add to the deterministic model of the SINS error, accelerometer and gyro white noise components. In addition, we add some white measurement noise to the measured velocity components. In accuracy of estimation, the stable value of $\psi_{z}$ is about $6^{\prime}$. It is due to unobservable state $\varepsilon_{x}$, but the estimation accuracy of $\psi_{x}$ and $\psi_{y}$ is very high. In order to accelerate the initial alignment, the convergence rate of $\psi_{z}$ must be increased.

\section{References:}

[1] Pinson, J. C. (1963), "Inertial guidance for cruise vehicles". In C.T. Leondes (Ed.), Guidance and control of Aerospace vehicle. New York; McGeaw-Hill, 1963, ch. 2

[2] Britting, K.R.(1971), "Inertial navigation System Analysis", New Yoek: Wiley-Interscince, 1971.

[3] Kayton, M., and Fried, W.R. (1969), “Avionics Navigation Systems”, New York: Wiley, 1969, ch.7.

[4] Siouris, G.M. (1993), “Aerospace Avionics Systems: A Modern Synthesis”, New York: Acadimic Press, 1993.

[5] Canon, R.H., Jr. (1961), "Alignment of inertial guidance systems by Gyrocompassing-linear theory", Journal of the Aerospace Science, Vol. 28, No. 11 (Nov.1961).

[6] Hung, J. C., and Witer, H. (1975), "Self-alignment techniques for inertial measurement units", IEEE Transaction on Aerospace and Electronics Systems, AES-11, 6 (Nov.1975), 1232-1247.

[7] Stieler, B., and Winter,H.(1982), "Gyroscopic instruments and their application to flight testing", AGARDDogrph 160, 15 (1982).

[8] Jiang, and Lin (1993), "Error Estimation of INS ground Alignment", IEEE Transaction On Aerospace and Electronic Systems Vol. 28, No.1 (jun.1993). 
[9] Bar-Itzhack, I.Y., Berman, N. (1988), "Control theoretic approach to inertial navigation systems", Journal of guidance, Control and dynamics, Vol. 11, No. 3 (May-June 1988), 237-245.

[10] Friedland, B. (1987), "Control System Design, An Introduction to state-space Methods", New York: McGraw-Hill, 1987.

[11] Huddle, J.R. (1983), "Inertial navigation system error model consideration in kalman filter applications", In C.T. Leondes (Ed.), Control and Dynamics Systems. New York: Acadimic press, 20 (1983).

[12] Meskin, D.G., and Ber-Itzhack,I.Y. (1992), "Observability analysis of piece-wise constant systems-part II: Application to inertial navigation in flight alignment" IEEE Transaction On Aerospace and Electronic Systems 28, 4(Oct.1992), 1068-1075.

[13] Jiang Fang and De Wan 1999, "A Fast Initial Alignment Method for SINS on Stationary Base", IEEE Transaction On Aerospace and Electronic Systems Vol. 32, No. 4 (Oct.1999), 1068-1075. 